Guy Keryer Éric Bailly

\section{Spécificité de l'action des protéine kinases et phosphatases dans la cellule}

Les kinases et les phosphatases sont généralement des structures modulaires comportant un domaine catalytique responsable de l'activité enzymatique et un domaine régulateur capable de contrôler cette activité. Dans le cas d'enzymes multimériques, chacun des domaines est porté par des sous-unités distinctes. La spécificité d'action des kinases et phosphatases au sein de la cellule résulterait : (1) de l'existence d'une grande diversité dans les sousunités régulatrices pour une même sous-unité catalytique et (2) du ciblage de la sous-unité catalytique vers ses substrats par la sous-unité régulatrice, assurant à cette dernière une double fonction. La sous-unité régulatrice et de ciblage serait, elle-même, la cible d'autres kinases ou phosphatases, multipliant ainsi les niveaux de régulation de ces enzymes. Les protéine kinases et phosphatases sont impliquées dans la transmission des signaux à et dans la cellule, et contrôlent en particulier le cycle cellulaire. De ce fait, leurs sous-unités régulatrices et de ciblage constituent des cibles potentielles de thérapeutiques antiprolifératives.

\section{ADRESSES}

G. Keryer : chargé de recherche au cors. Laboratoire de physiopathologie du développement, École normale supérieure, 46, rue d'Ulm, 75230 Paris Cedex 05, France. E. Bailly: chercheur post-doctoral. Department of molecular biology, MB7, the Scripps research institute, $10666 \mathrm{~N}$. Torrey Pines

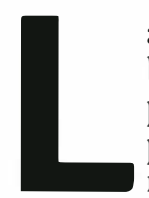

a phosphorylation réversible des protéines, catalysée par les kinases et les phosphatases, est un des mécanismes post-traductionnels les plus utilisés par les cellules eucaryotes pour contrôler des processus biologiques aussi variés que le métabolisme, la transmission des signaux extracellulaires, l'expression génétique ou encore les différentes étapes du cycle cellulaire. Toutefois, un grand nombre de ces enzymes présentent, in vitro, une large spécificité de substrats: cela ne reflète pas nécessairement leur activité, plus restreinte au sein de la cellule, et qui intervient généralement en réponse à un signal spécifique. A la lumière de nombreuses études, la localisation des protéine kinases et phosphatases à proximité de leurs 
substrats apparaît comme un facteur essentiel de spécificité pour ce type de réaction enzymatique. Cette localisation préférentielle serait assurée par une interaction entre l'enzyme et le substrat lui-même, ou entre l'enzyme et un composant cellulaire associé au substrat. Cela suppose donc une association directe entre une partie de l'enzyme, ou l'un des éléments du complexe enzymatique, et les composants cellulaires en question. Un grand nombre de protéine sérine/thréonine kinases et phosphatases sont constituées de deux sous-unités: l'une porte la fonction catalytique, l'autre la fonction de régulation. Ce serait cette dernière qui permettrait également la localisation spécifique de la par- tie catalytique. Elle assurerait donc une double fonction de régulation, de l'activité d'une part, et de la position de l'enzyme vis-à-vis des substrats d'autre part. Il en résulterait une modulation plus fine de l'activité enzymatique, et des niveaux supplémentaires de régulation pour une même enzyme.

Originellement proposée par Rubin, Erlichman et Scott pour la famille des kinases dépendantes de l'AMPc [1], l'idée de protéines régulatrices associées à la sous-unité catalytique de ces kinases et dirigeant ces dernières vers leurs substrats cellulaires spécifiques, s'étend maintenant à un grand nombre d'autres kinases et phosphatases [2].

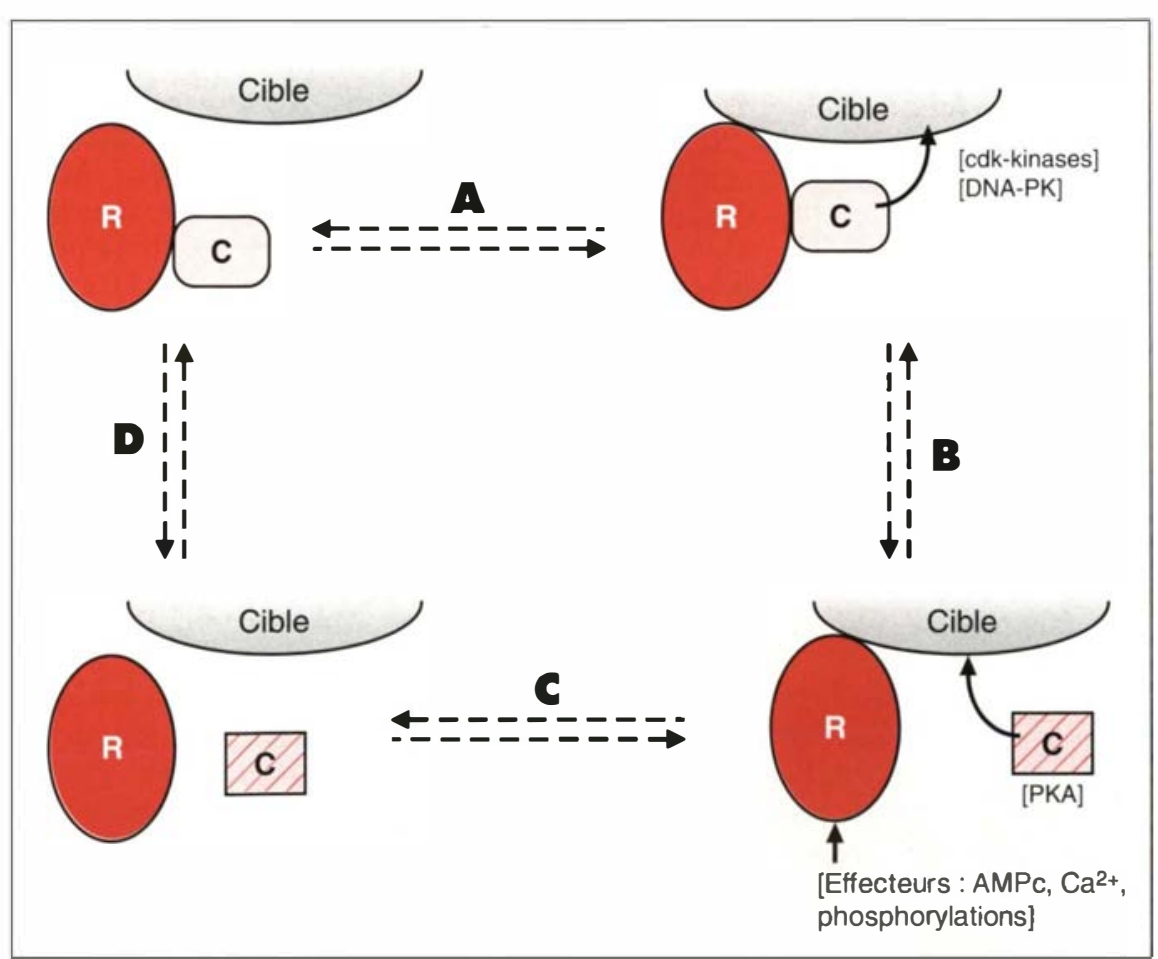

Figure 1. Le modèle du ciblage d'un grand nombre de protéine kinases phosphorylant ou déphosphorylant une sérine ou thréonine. A. Le complexe $R-C(R=$ sous-unité régulatrice et de ciblage; $C=$ sous-unité catalytiquel peut s'associer à un organe cible, privilégiant l'action de $C$ sur des protéines de cette cible. C peut agir, soit complexée à $R$ (cas des cdk kinases où $R=$ cyclines et de la DNA activated protein kinase où $R=k u[28]$ ), soit B : dissociée de $R$ (cas des PKA) après action d'un effecteur sur cette dernière sous-unité. C. L'association R-cible peut être rompue après phosphorylation de la cible ou de $R$ par diverses kinases. D. Une fois libre $R$ peut ou non se réassocier à $C$. Toutes ces réactions peuvent être réversibles.

\section{Cibler les sous-unités régulatrices des kinases et phosphatases}

Les kinases et les phosphatases sont généralement des structures modulaires comportant un domaine responsable de l'activité enzymatique et un domaine capable de contrôler positivement ou négativement cette activité. Dans le cas des kinases et phosphatases multimériques, auxquelles nous nous limiterons volontairement par souci de simplicité, chacun des domaines est porté par des protéines ou sous-unités distinctes. Ce type d'organisation permet une combinatoire des différentes sous-unités, combinatoire qui est d'autant plus importante que les isoformes de chacune des sous-unités sont nombreuses. Ainsi, à un type de sous-unité catalytique (C) peuvent s'associer un ou plusieurs types de sous-unité régulatrice $(R)$ et réciproquement. Dans l'exemple des kinases dépendantes des cyclines sur lequel nous reviendrons, une même sous-unité catalytique de la kinase mitotique, la sous-unité p34 ${ }^{\text {ddr } 2}$, peut s'associer aux cyclines A ou B. Inversement, une même sous-unité régulatrice, la cycline A, peut interagir avec des sous-unités catalytiques différentes, la p34 atc2 ou la p33 $3^{\text {rtk2 }}$. A côté de sa fonction régulatrice proprement dite, $\mathrm{R}$ semble avoir un autre rôle, celui de diriger $\mathrm{C}$ vers son ou ses substrats. De nombreuses études portant sur différentes kinases et phosphatases suggèrent en effet que la spécificité de substrat de ces enzymes est assurée par une interaction directe entre $\mathrm{R}$ et une protéine cible, qui peut être ellemême le substrat ou associée au substrat (figure 1).

L'intérêt de ce mécanisme de ciblage serait de moduler l'accessibilité de C vis-à-vis de ses différents substrats ou inhibiteurs potentiels. Un autre avantage est d'introduire des niveaux de régulation supplémentaires.

Les trois principales caractéristiques de ce modèle sont : une localisation préférentielle de la sous-unité catalytique; la régulation de l'activité de cette sous-unité catalytique ; la réversibilité des interactions entre $\mathrm{C}, \mathrm{R}$ et les cibles, permettant ainsi de trans- 


\section{RÉFÉRENCES}

1. Scott JD, Carr D. Subcellular localization of the type II cAMP-dependent protein kinase. News Physiol Sci 1992; 7 : 143-8.

2. Hubbard MJ, Cohen P. On target with a new mechanism for the regulation of protein phosphorylation. Trends Biochem Sci 1993 ; 18: 172-7.

3. Taylor SS, Buechler JA, Yonemoto W' cAMP-dependent protein kinase: framework for a diverse family of regulatory enzymes. Annu Rev Biochem 1990; 59: 971-1005.

4. Ratoosh SL, Richards JS. Regulation of the content and phosphorylation of RII by adenosine 3',5'-monophosphate, folliclestimulating hormone and estradiol in cultured granulosa cells. Endocrinology 1985; 117: 917-27.

5. Tortora G, Clair T, Katsaros D, et al. Induction of megakaryocytic differentiation and modulation of protein kinase gene expression by site selective cAMP analogs in K-562 human leukemic cells. Proc Natl Acad Sci USA 1989; 86: 2849-52.

6. Carr D, Scott JD. Blotting and bandshifting: techniques for studying proteinprotein interactions. Trends Biochem Sci $1992 ; 17: 246-9$.

7. Ndubuka C, Li Y, Rubin C. Expression of A kinase anchor protein 75 depletes type II cAMP-dependent protein kinases from the cytoplasm and sequesters the kinases in a particulate pool. J Biol Chem $1993 ; 268: 7621-4$.

8. Keryer G, Cavadore JC, Bornens M. Mitotic phosphorylation of the regulatory subunit of cAMP-dependent protein kinase type II by cdc2 kinase. J Cell Biol 1991; 115: 284a.

* Cel article utilise le code à une lettre des acides aminés. A : Ala; C: Cys ; D:Asp; E: Glu;F: Phe; G: Gly; H: His; 1: Me; K: L.ys; L.: Leu, $M:$ Met $; N: A s n ; P:$ Pro; $Q: G l n ; R: A r g$; $S:$ Ser; T: Thr; V: Val; W: Trp; X représente férer rapidement l'activité de C d'un compartiment à un autre.

Pour illustrer le modèle proposé, nous avons choisi deux exemples parmi les sérine/thréonine protéine kinases, les protéine kinases dépendantes de l'AMPc ou PKA et les protéine kinases dépendantes des cyclines ou cdk, et un exemple de sérine/thréonine protéine phosphatase, la phosphatase de type 1 ou PP1. La localisation de ces trois protéines en des sites cellulaires particuliers ou dans des complexes protéiques bien précis peut expliquer l'action restreinte de ces enzymes in situ.

\section{Une sous-unité catalytique similaire ciblée par de nombreux isotypes de sous-unités régulatrices}

Dans les cellules de mammifères, les PKA constituent une famille d'enzymes dont chaque holoenzyme correspond au complexe $\mathrm{R}_{\mathrm{i}} 2 \mathrm{C}$, associant deux sous-unités catalytiques (C) à un dimère de sous-unités régulatrices $\left(R_{2}\right)\left[\begin{array}{ll}1, & 3\end{array}\right]$. Sous l'influence de signaux divers (hormonaux, nerveux, etc.), l'activité de l'adénylyl cyclase couplée au récepteur correspondant est stimulée. Il en résulte une élévation de la concentration en AMPc, lequel peut agir sur une PKA proche de l'adénylyl cyclase, mais aussi diffuser et se concentrer dans différents compartiments subcellulaires. Une compartimentation de l'AMPc, induite par diverses hormones, a été proposée [1]. L'augmentation de la concentration en AMPc est responsable de la levée de l'inhibition de C par R, par dissociation du complexe $\mathrm{R}_{2} 2 \mathrm{C}$ selon la réaction:

$\left(\mathrm{R}_{2} 2 \mathrm{C}\right)_{\text {inacuif }}+4$ AMPc $\rightarrow$ AMPc-R2 $+2 \mathrm{C}_{\text {actif }}$

Trois isoformes de sous-unités catalytiques $(C \alpha, C \beta$ et $C \gamma)$, ont été décrites, fortement analogues entre elles. Il existe deux types de sous-unité régulatrice, RI et RII, sur la base desquels la nomenclature des PKA a été établie (PKA de type I et de type II). Enfin, à chaque type de $\mathrm{R}$ correspondent deux gènes $\alpha$ et $\beta$, portant à quatre le nombre d'isoformes pour la sous-unité régulatrice des PKA. RI et RII se distinguent par des différences importantes dans leur région $\mathrm{N}$-terminale, ainsi que par la présence, dans le cas de RII, d'un site de phosphorylation par C (motif $\left.\mathrm{RR}^{\mathrm{A}} / \mathrm{VS}^{*}\right)$. La phosphorylation de cette sérine, située dans le domaine d'interaction avec C, entraîne une diminution de l'affinité de RII pour $\mathrm{C}$ et donc une dissociation de l'holoenzyme à basse concentration d'AMPc.

Les sous-unités régulatrices $\mathrm{RI} \alpha$ et RII $\alpha$ sont présentes dans la plupart des tissus. L'expression de RI $\beta$ et RII $\beta$ semble beaucoup plus restreinte. Bien qu'initialement décrite comme spécifiquement neuronale, RII $\beta$ a pu être mise en évidence dans de nombreux autres tissus et cellules en culture, avec toutefois un niveau d'expression beaucoup moins élevé que celui observé dans le cerveau. A cette expression différentielle correspond une régulation génique spécifique de chacune des isoformes de $\mathrm{R}$, à savoir, constitutive pour certaines comme RII $\alpha$, et inductible pour d'autres. Ainsi, l'expression de RII $\beta$ est sélectivement induite lors de la stimulation hormonale des cellules de la granulosa ovarienne [4] et au cours de l'induction de la différenciation des mégacaryocytes par l'AMPc [5].

Contrairement à RI qui est principalement cytosolique, chacune des isoformes de RII est majoritairement exprimée sous une forme particulaire. De nombreuses études biochimiques et immunocytochimiques montrent que la localisation subcellulaire des PKA de type II est assurée par la propriété de RII de se lier fortement à un certain nombre de protéines du cytosquelette et membranaires. Les auteurs anglosaxons ont proposé le terme AKAP pour A kinase anchoring protein, désignant ainsi les protéines capables d'interagir avec RII et permettant l'ancrage des PKA de type II. De fait, l'essentiel des protéines liant RII, caractérisées jusqu'à présent, sont associées aux membranes ou au cytosquelette. Cependant, il semble que des protéines également capables de lier RI mais présentes dans le cytosol existent aussi dans les cel- 


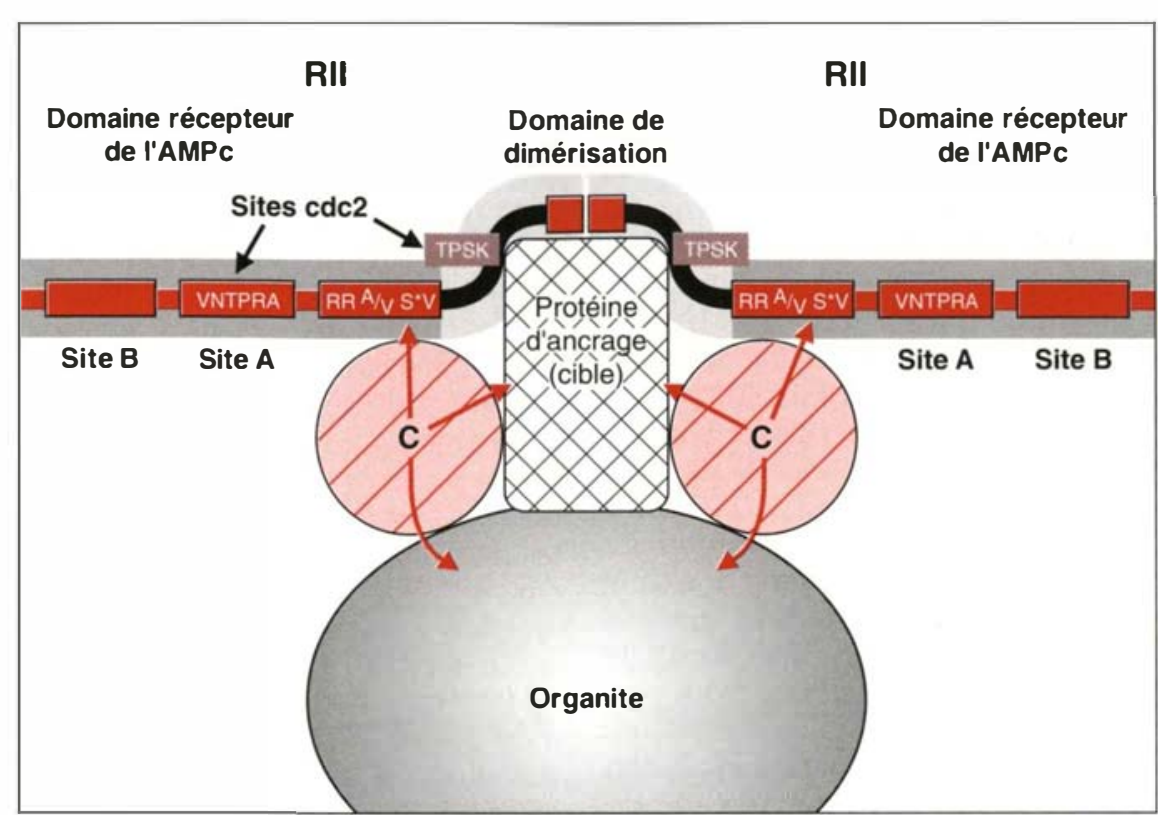

Figure 2. Un modèle pour l'interaction entre la PKA de type II et les cibles de cette enzyme. La sous-unité RIl possède quatre domaines: de la partie $\mathrm{N}$-terminale vers l'extrémité C-terminale on y distingue le domaine de dimérisation, ce domaine et le domaine suivant étant nécessaires aux interactions de Rll avec les AKAP (A kinase anchoring proteins); un domaine d'interaction avec la sous-unité catalytique $C$ et enfin le domaine récepteur portant les deux sites de liaison de l'AMPc. Au moins trois domaines comprennent dans leur séquence un site de phosphorylation. Le domaine d'interaction avec les AKAP présente deux à trois sites pour des kinases comme la caséine kinase II, la glycogène synthase 3, les cdk kinases. Le domaine d'interaction avec la sous-unité catalytique $C$ est phosphorylé par elle sur un site consensus RRXS*. Enfin, le premier domaine de liaison à l'AMPC porte un site potentiel de phosphorylation pour les cdk kinases. $S^{*}$ : Sérine phosphorylée.

lules de mammifères. Aussi serait-il peut-être plus judicieux d'utiliser le terme plus général AKIP pour $A$ kinase interacting protein pour désigner l'ensemble des cibles de R. L'interaction de RII avec ses différentes cibles nécessite la dimérisation de RII. Elle fait aussi intervenir un domaine NH2-terminal de RII comprenant les acides aminés 30 à 80 (figure 2). Les analyses de séquences montrent que ce domaine d'interaction correspond à la région la plus variable des isoformes de RII. Cette variabilité pourrait donc refléter la diversité d'interaction de RII ces de délétion partielle ont permis d'attribuer à la région C-terminale de ces protéines un rôle déterminant dans leur propriété de se lier à RII, bien qu'aucune analogie de séquence n'apparaisse de façon claire dans cette région. La prédiction de structures secondaires rend fortement probable une organisation en hélice $\alpha$ amphipathique (hélice $\alpha$ comportant une face polaire et une face apolaire opposée). Il est à souligner que deux de ces protéines d'ancrage sont également capables d'interagir avec la calmoduline par leur partie N-terminale, et pourraient donc servir de carrefour entre la voie de signalisation dépendante de l'AMPc et la voie dépendante du calcium. Dans les dendrites, la localisation préférentielle de telles protéines, près du lieu de réception des signaux nerveux, constitue un autre argument en faveur de cette hypothèse. Plusieurs banques d'expression ont été criblées par la technique d'overlay des protéines. Cette technique [6] est une modification de la technique de Western blot. Après la séparation électrophorétique des protéines et leur transfert sur membrane de nitrocellulose, les AKAP sont repérées par autoradiographie après incubation du filtre avec de la sous-unité RII (protéine entière ou tronquée, isolée à partir de tissus animaux ou surexprimée dans la bactérie), préalablement phosphorylée en présence de phosphore ${ }^{32} \mathrm{P}$ par la sous-unité catalytique $\mathrm{C}$. Cette technique a déjà permis d'identifier plus de 30 protéines d'ancrage potentielles, capables de lier RII dans différents tissus bovins et humains. Une même isoforme de RII ( $\alpha$ ou $\beta$ ) peut se lier à différentes AKAP présentes dans différents compartiments subcellulaires. C'est en fait la spécificité de l'AKAP qui définit la présence ou non de ce type de PKA. La surexpression de l'AKAP 75 bovine, associée au cytosquelette des dendrites neuronaux, altère la distribution particulaire/cytoplasmique de la PKAII $\beta$ [7].

Par ailleurs, la phosphorylation de $\mathrm{R}$ sur différents sites (voir figure 2) apporte un niveau supplémentaire de régulation de l'activité de la PKA. La sous-unité régulatrice RII possède 


\section{RÉFÉRENCES}

9. Keryer (;, Luo Z, Cavadore J C, Erlichman J, Bornens M. Phosphorylation of the regulatory subunit of type RII $\beta$ cAMPdependent protein kinase by cyclin $\mathrm{B} / \mathrm{p} 34^{\text {ddc2 }}$ kinase impairs its binding to microtubule-associated protein 2. Proc Natl Acad Sci L'SA 1993; 90): 5418-22.

10. Cohen P. The structure and regulation of protein phosphatases. Annu Rer Biochem 1988 ; 58 : 453-508.

11. Dorée M. Le complexe cdc2-cycline: un facteur universel pour l'entrée en initose. médecine/sciences $1990 ; 6: 8-9$.

12. (Girard F, Lamb N, Fernandez A. Le cycle cellulaire analysé par microinjection dans les cellules somatiques : rôles distincts des cyclines A et B. médecine/sciences 1992 ; 8: $375-8$.

13. Pines J, Hunter T. Human cyclins A and $\mathrm{Bl}$ are differentially located in the cell and undergo cell cycle dependent nuclear transport. I Cell Biol 1991; 115: 1-17.

14. Bailly E, Pines J, Hunter T, Bornens M. Cytoplasmic accumulation of cyclin B 1 in human cells: association with a detergent-resistant compartment and with the centrosome. I Ciell $S_{c i} 1992 ; 101$ : 529-45.

15. Ookata K, Hisanaga S, Okumura E, Kishimoto T. Association of p.34 cdr2/cyclin B complex with microtubules in Starfish oocytes. / Ciell Sa 1993; 105: 873-81.

16. Karsenti E. Vers une description du mécanisme d'assemblage du fuseau mitotique à l'échelle moléculaire. médecine/sciences 1993; 9: 131-9.

17. Lamas E, Zindy F, Sobczack J, Paterlini $\mathrm{P}, \mathrm{W}$ ang J, Chenivesse $\mathrm{X}$, Henglein $\mathrm{B}$, Bréchot C. Cycline A et cancer. médecine/sciences $1993 ; 9$ : 676-83.

18. Peeper DS, Parker LL, Ewen ME, el al. A- and B- type cyclins differentially modulate substrate specificity of cyclin-cdk complexes. EMBO J 1993; 12 : 1947-54. en effet plusieurs sites potentiels de phosphorylation, à la fois dans le domaine de réception de l'AMPc (site A), dans le domaine d'interaction avec la sous-unité catalytique déjà mentionnée (RRXS*) et dans le domaine $\mathrm{N}$-terminal d'interaction avec les protéines d'ancrage. La phosphorylation du site RRXS* affecte l'interaction R-C. La phosphorylation in vitro de RII $\alpha$ par la kinase mitotique cdc2-cycline $B$ modifie le degré d'interaction entre RII et C de telle sorte qu'une concentration plus élevée d'AMPc est nécessaire pour dissocier le complexe $\mathrm{R}_{2} 2 \mathrm{C}$ [8]. La phosphorylation de RII peut aussi modifier son interaction avec les cibles de la PKA de type II. Récemment, nous avons montré que la phosphorylation de RII $\beta$ au niveau de son domaine d'interaction avec MAP-2 par le

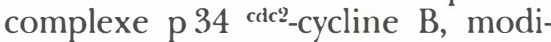
fie l'interaction entre ces deux protéines [9]. Ainsi, la phosphorylation multiple de RII pourrait avoir des répercussions fonctionnelles importantes, tant sur l'activité de C, en rendant l'holoenzyme moins sensible à l'AMPc, que sur sa localisation subcellulaire, en interférant avec ses propriétés d'interaction avec les protéines d'ancrage.

Enfin, il est singulier de constater que, dans le muscle cardiaque, RII est elle-même une cible probable de la phosphatase de type 2B (PP2B, [10]), dont l'activité est contrôlée par le calcium via la calmoduline. La PP2B est co-purifiée, au moins partiellement, avec RII. La déphosphorylation du site $\mathrm{RR}^{\star} / \mathrm{V}^{*}$ de RII par cette phosphatase favorise la réassociation de l'holoenzyme, conduisant ainsi à l'inactivation de C. Le caractère antagoniste bien connu du calcium vis-à-vis des effets de l'AMPc s'explique donc, non seulement par le fait que nombre de substrats de la PP2B sont aussi substrats de la PKA, mais aussi par un effet inhibiteur direct de la PP2B sur la sous-unité régulatrice des PKA de type II.

Si, sur le plan conceptuel, on peut imaginer que la phosphorylation de cibles par la PKA ou d'autres kinases puisse également altérer les propriétés d'ancrage de ces protéines ou leurs propriétés de fixation à d'autres effecteurs, aucun argument expérimental direct ou indirect n'est encore venu confirmer une telle hypothèse.

\section{Différentes sous-unités catalytiques ciblées par différentes sous-unités régulatrices : l'exemple des cdk}

Un autre exemple de kinases fonctionnant sur un mode de ciblage analogue est celui des kinases dépendantes des cyclines ou cdk, dont l'activation gouverne la progression du cycle cellulaire chez les eucaryotes supérieurs $\left(m / s \quad n^{\circ} 1\right.$, vol. 6, p. 8). Cette famille de kinases est composée d'hétérodimères comprenant une sous-unité catalytique, cdk, et une sous-unité régulatrice, appelée cycline. Les cyclines ont d'abord été identifiées dans les embryons précoces d'oursins et d'étoiles de mer grâce à leur accumulation en interphase et leur destruction lors de chaque mitose. Ainsi, le rôle des cyclines A et B dans l'activation de la protéine kinase $\mathrm{p} 34^{\text {ddc2 }}$ (cdk1), la première cdk caractérisée à partir de mutants du cycle cellulaire de levure, et dans l'initiation de la phase $M$ qui en résulte, est aujourd'hui particulièrement bien documenté [11, 12].

L'hypothèse d'un mécanisme de ciblage concernant cette famille de protéine kinases a été initialement proposée pour essayer de rendre compte des observations paradoxales suivantes: bien qu'il soit, au moins dans certains cas, possible de les distinguer fonctionnellement les unes des autres, toutes ces kinases de la famille des cdk présentent in vitro les mêmes spécificités de substrat. Un premier argument en faveur de l'hypothèse du ciblage a été apporté par l'observation d'importantes différences dans la distribution intracellulaire des cyclines. Dans les cellules somatiques, la cycline A s'accumule principalement dans le noyau, contrairement à la cycline $\mathrm{B}$ dont l'accumulation plus tardive est restreinte au cytoplasme, celle-ci ne devenant nucléaire que juste avant la rupture de l'enveloppe nucléaire en début de prophase [13, 14]. Même si les interactions sont diffé- 
rentes de celles mises en jeu dans le cas des protéines qui lient RII, des résultats obtenus au laboratoire suggèrent, par ailleurs, l'existence d'un phénomène d'ancrage du complexe $\mathrm{p} 34^{\text {rttr }}$-cycline $\mathrm{B}$ analogue à celui de la PKA. C'est ainsi que plus de $90 \%$ de la cycline B s'accumule dans les cellules humaines sous une forme qui résiste à l'extraction par les détergents non ioniques. Il est intéressant de noter ici qu'une interaction entre la cycline $\mathrm{B}$ et la protéine associée aux microtubules MAP4 a été décrite par plusieurs groupes [15]. MAP4 pourrait donc bien être la première cible identifiée de la cycline B et être ainsi responsable, au moins en partie, de l'ancrage du complexe p34 ${ }^{\text {dd } 2}$-cycline $\mathrm{B}$, dans le cytoplasme des cellules interphasiques, et au niveau du fuseau des cellules en mitose. D'un point de vue fonctionnel, l'importance d'un tel phénomène de ciblage de la p34 ddr via la cycline B a déjà été discuté dans le cadre des mécanismes fondamentaux d'assemblage du fuseau mitotique [16].

D'autres membres de la famille cdk sont impliqués dans le contrôle de la transition Gi/S. L'exemple le mieux documenté à cet égard est celui de la $\mathrm{p} 33^{\text {rk2 }}$, la cdk structurellement la plus proche de la p34 $4^{\text {rdtr }}$. Des travaux récents attribuent aux complexes p33 ${ }^{\text {cdk2 }}$-cycline $\mathrm{E}$ et p33 ${ }^{\text {rkz2 }}$-cycline A des rôles déterminants, respectivement dans l'initiation et la progression de la phase S [17]. De façon surprenante, on constate à nouveau qu'en dépit de caractéristiques fonctionnelles distinctes ces deux types de complexes ont manifestement des cibles communes, à savoir le facteur de transcription E2F ainsi que la protéine p107 apparentée à la protéine p105, codée par le gène suppresseur de tumeur du rétinoblastome $\mathrm{Rb}$. Des

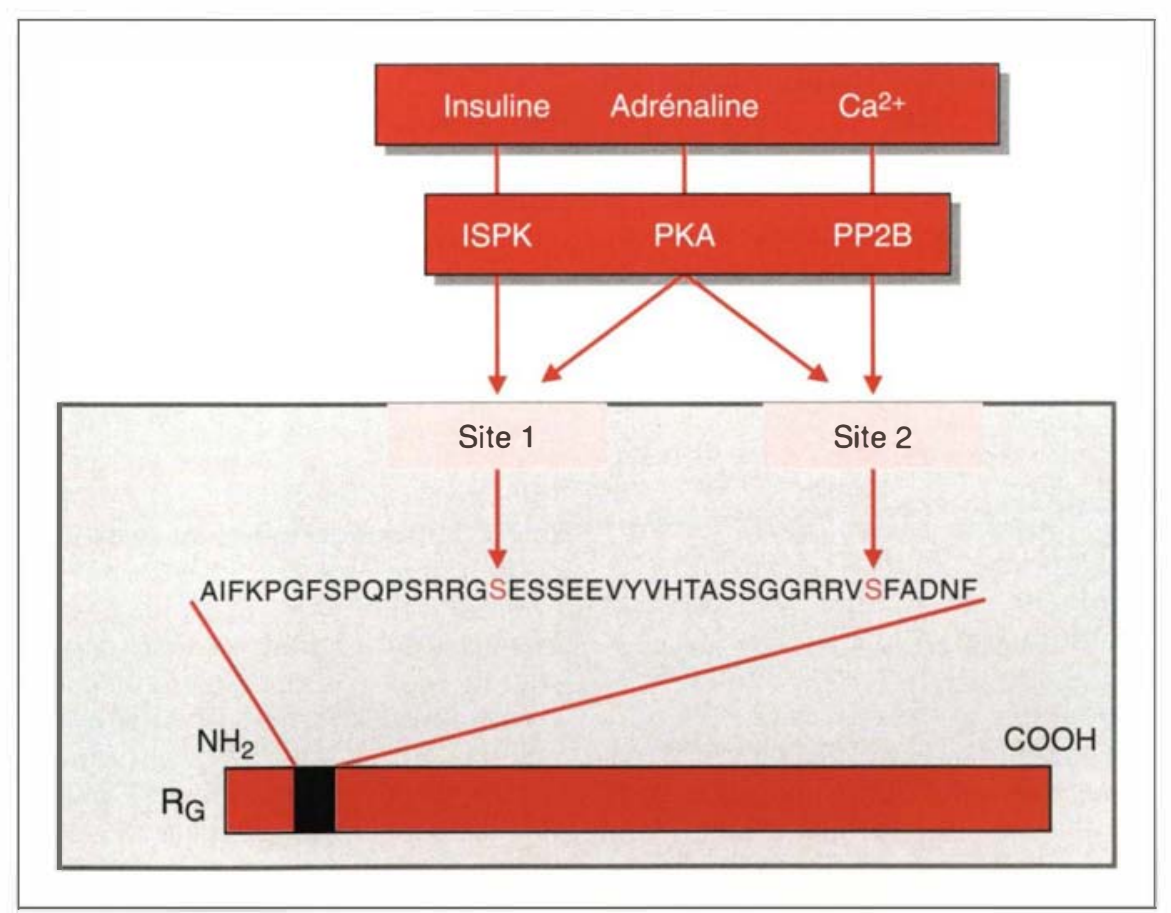

Figure 3. Régulation de l'activité de la phosphatase de type 1 (PP1G) via la phosphorylation réversible des sites RRXS* présents sur la sous-unité régulatrice $R_{G}$ par la protéine kinase stimulée par l'insuline (ISPK), par la kinase dépendante de l'AMPc (PKA) et la protéine phosphatase dépendante du Caª $\mathrm{Ca}^{2}$ PP. résultats récents [18] font clairement ressortir que c'est la sous-unité de cycline A qui joue un rôle critique dans le ciblage in vivo de la partie catalytique p33 ${ }^{\text {rctk2 }}$ vers la pl07. Il est probable qu'à la diversité des cyclines correspond une diversité de cibles: leur identification permettra de mieux comprendre la fonction propre à chacune de ces kinases.

\section{La phosphatase de type 1}

Comme dans le cas des réactions de phosphorylation, il semble que la cellule ait adopté une stratégie analogue pour dicter aux phosphatases leur spécificité d'action [10, 19]. Le cas de la sérine/thréonine phosphatase de type l (PPl) en fournit une bonne illustration. L'activité de cette enzyme est portée par une sousunité catalytique ( $\mathrm{PPl}_{\mathrm{C}}$ ) de $37 \mathrm{kDa}$. In vitro cette sous-unité est capable de déphosphoryler un grand nombre de substrats phosphorylés in vivio par différentes kinases.

Le premier exemple de ciblage de la $\mathrm{PPl}_{\text {c: }}$ provient de l'isolement, à partir de muscle squelettique, d'une forme de $\mathrm{PPl}$ associée au glycogène $\left(\mathrm{PPl}_{c_{i}}\right)$. La PPl ${ }_{(;}$est un hétérodimère constitué de $\mathrm{PPl}_{\mathrm{C}}$ et d'une sous-unité régulatrice de $124 \mathrm{kDa}$ associée au glycogène, que nous appelerons $R_{i}$. Les substrats de la $\mathrm{PPl}_{\text {; }}$ ne sont pas le glycogène luimême, mais trois enzymes multimériques, impliquées dans le métabolisme de celui-ci : la glycogène phosphorylase, la phosphorylase kinase et la glycogène synthétase. Ces trois enzymes sont elles-mêmes associées au glycogène par l'intermédiaire de sous-unités régulatrices. L'activité phosphatase de la $\mathrm{PPl}_{G}$ envers la phosphorylase associée au glycogène est huit fois plus élevée que celle de la $\mathrm{PPl}_{\mathrm{C}}$ : cela témoigne de l'importance de ce processus de ciblage de la $\mathrm{PPl}_{\mathrm{C}}$ au glycogène dans la régulation des trois enzymes en question. En outre, la spécificité de cette interaction fonctionnelle avec le glycogène est attestée par le fait que la $\mathrm{PPl}_{\text {; }}$, ne montre une activité augmentée que vis-à-vis de substrats liés au glycogène.

Comme dans le cas de la sous-unité régulatrice RII de la PKA, l'état de phosphorylation de la sous-unité 


\section{RÉFÉRENCES}

19. Shenolikar S, Nairn AC. Protein phosphatases: recent progresses. Adv Second Messenger Phosphoprotein Res 1991; 23: 1-121.

20. Stone EM, Yamano $\mathrm{H}$, Kinoshita $\mathrm{N}$, Yanagida M. Mitotic regulation of protein phosphatases by the fission yeast sds22 protein. Curr Biol 1993; 3: 13-26.

21. Vandré DD, Borisy GG. The centrosome cycle in animal cells. In yam JS, Brinrley BR, eds. Mitosis: molecules and mechanisms. San Diego: Academic Press, $1989: 39-75$

22. Glover DM, Cayetano G, Raff JW. Le centrosome. Pour la srience 1993; 190 : 50-8.

23. Nigg EA., Schafer G, Hiltz H, Eppenberger HM. Cyclic-AMP-dependent protein kinase type II is associated with the Golgi complex and with centrosomes. Cell 1985; 41: 1039-51.

24. De Camilli P, Moretti P, Denis-Donini S, Walter U, Lohmann SM. Heterogeneous distribution of the cAMP receptor protein RII in the nervous system: evidence for its intracellular accumulation on microtubules, microtubule-organizing centers and in the area of the Golgi complex. J Cell Biol $1986 ; 103$ : 189-203.

25. Keryer G, Rios RM, Landmark BF, Skalhegg B, Lohmann SM, Bornens M. A high affinity binding protein for the regulatory subunit of cAMP-dependent protein kinase II in the centrosome of human cells. Exp Cell Res 1993; 204: 230-40.

26. Bailly E, Dorée M, Nurse P, Bornens M. p34alc2 is located in both nucleus and cytoplasm ; part is centrosomally associated at $\mathrm{G} 2 / \mathrm{M}$ and enters vesicles at anaphase. EMBO J 1989 ; 8 : 3985-95.

27. Brewis ND, Street AJ, Prescott AR, Cohen PTW. PPX a novel protein serine/threonine phosphatase localized to centrosomes. EMBO J 1993; 12: 987-96.

28. Anderson CW. DNA damage and the DNA-activated protein kinase. Trends Bio- régulatrice de la $\mathrm{PPl}_{\mathrm{G}}$, permet d'intégrer, dans la régulation de cette phosphatase, l'action de plusieurs voies de signalisation (AMPc, calcium, etc.). Les sites de phosphorylation de $R_{r}$; sont au nombre de deux et correspondent à deux sérines situées près de l'extrémité $\mathrm{N}$ terminale de la protéine (figure 3). La phosphorylation du premier site par la kinase dépendante de l'insuline (ISPK), ou par la PKA, augmente l'activité de la $\mathrm{PPl}_{G}$, vis-à-vis de la glycogène synthétase et de la phosphorylase kinase, favorisant ainsi la synthèse de glycogène. La phosphorylation de l'autre site par la PKA entraîne la libération de la sous-unité catalytique dans le cytosol. Il en résulte une inhibition de l'enzyme, inhibition qui provient à la fois de la perte de l'action du glycogène sur la $\mathrm{PPl}_{G}$ et de la sensibilité accrue de la $P P l_{c}$ ainsi relarguée aux inhibiteurs protéiques présents dans le cytosol. La déphosphorylation de $R_{f}$, par la phosphatase dépendante du calcium PP2B conduit à la réassociation et, par voie de conséquence, à la réactivation de $\mathrm{PPl}_{C}$. Le rôle potentiel de la PP2B serait de permettre une modulation combinée des voies de transmission des signaux impliquant calcium et AMPc.

L'existence de cibles multiples pour une même sous-unité régulatrice se rencontre également dans le cas de la $\mathrm{PPl}_{\mathrm{G}}$. Dans le muscle strié, $\mathrm{R}_{\mathrm{q}}$ se trouve également fortement associée au réticulum sarcoplasmique qui intervient dans la régulation du pompage des ions calcium et la relaxation du muscle. Il est à noter que, dans ce cas, l'interaction se fait par l'intermédiaire d'un domaine hydrophobe situé à l'extrémité Cterminale de $R_{4}$; qui est différent du domaine d'interaction avec le glycogène situé en N-terminal de la protéine.

Le métabolisme du glycogène hépatique fait intervenir une sous-unité régulatrice $R_{I I}$, différente de celle qui vient d'être décrite dans le cas du muscle strié. De même, l'association entre la $\mathrm{PPl}_{\mathrm{c}}$ et les myofibrilles des muscles lisses fait appel à un troisième type de sous-unité régulatrice, composée de deux protéines de 130 et $20 \mathrm{kDa}$. Enfin, des obser- vations immunocytochimiques mettent en évidence un fort enrichissement de la PPl dans le noyau des cellules eucaryotes durant l'interphase, et au niveau des chromosomes lors de la mitose. Récemment, le groupe de Yanagida de l'université de Kyoto [20] a pu isoler un gène essentiel de Schizosaccharomyces pombe dont le produit de traduction correspond vraisemblablement à la sous-unité régulatrice d'une phosphatase de type I, impliquée dans la transition métaphase-anaphase.

Le mécanisme de ciblage d'une sous-unité catalytique par l'intermédiaire de la sous-unité régulatrice qui lui est associée semble donc être largement utilisé par la PPl pour contrôler des processus cellulaires aussi variés que le métabolisme du glycogène dans différents tissus, la contraction musculaire ou la régulation de certaines fonctions nucléaires. Il reste à déterminer si ce mécanisme est utilisé par d'autres phosphatases, analogues ou non de la PPl.

\section{Le centrosome, une cible privilégiée de ce type de kinases ou de phosphatases}

Plusieurs études d'immunocytochimie [21] font apparaître le centrosome comme étant un exemple de cible privilégiée de certaines kinases et phosphatases. Cet organite, composé de deux cylindres centriolaires et d'un matériel péricentriolaire dense aux électrons [22], a pour fonction principale la nucléation des microtubules des cellules interphasiques et du fuseau mitotique. La première kinase identifiée au niveau du centrosome fut la PKAII $\alpha$ [23-25] comme l'illustre l'accumulation de la sous-unité régulatrice RII $\alpha$ dans la région centrosome-Golgi observée par immunofluorescence dans des cellules HeLa (figure 4A). Un double marquage de l'appareil de Golgi et de la sous-unité régulatrice RII $\alpha$ révèle l'accumulation de cette sousunité au centrosome et à l'appareil de Golgi. En accord avec ces données d'immunofluorescence, l'holoenzyme est fortement enrichie dans des préparations de centrosomes isolés de lymphoblastes 


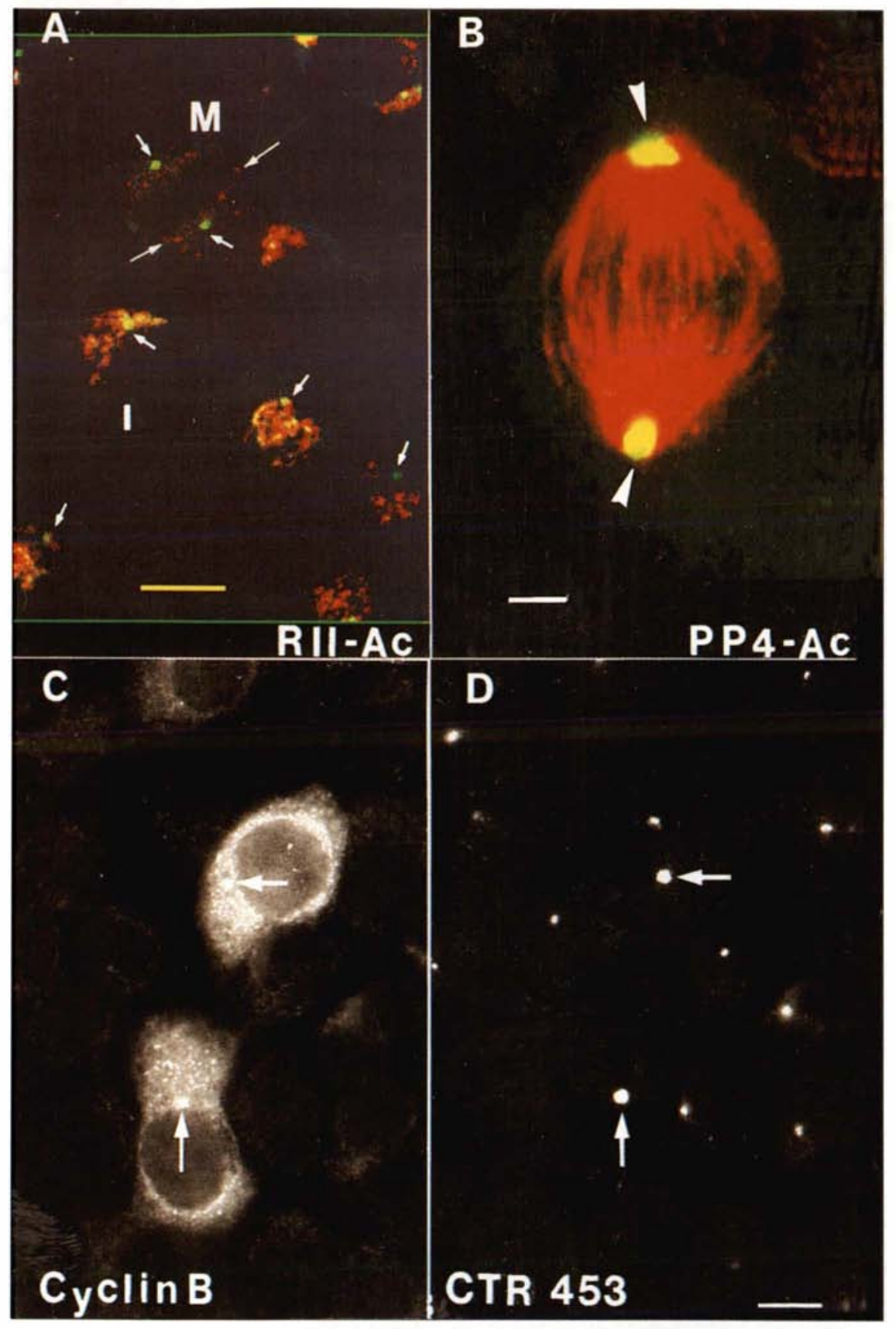

Figure 4. A. Double marquage en microscopie confocale de cellules HeLa avec un anticorps polyclonal dirigé contre la sous-unité régulatrice de la PKA II (en vert, RII-Ac) et un anticorps monoclonal dirigé contre une protéine golgienne (en rouge). 1: cellule en interphase; les centrosomes sont marqués par RII-Ac (petites flèches blanches). M : cellule en mitose; l'appareil de Golgi est éclaté (longues flèches blanches) et les pôles marqués par RII-Ac. Barre: $6 \mu \mathrm{m}$. B. Double marquage en microscopie confocale d'une cellule HeLa en mitose avec un anticorps dirigé contre la sous-unité catalytique de la phosphatase PPX ou PP4 (PP4-Ac, en vert) et un anticorps dirigé contre la tubuline (en rouge). La phosphatase PP4 est concentrée aux pôles du fuseau mitotique. Barre: $3,5 \mu \mathrm{m}$. C et D. Double marquage de cellules HeLa avec un anticorps polyclonal dirigé contre la cycline $B 1$ (C) et un anticorps monoclonal (CTR453) dirigé contre un antigène du centrosome (D). Les cellules en phase G2 montrent une accumulation de cycline $B 1$ au centrosome près du noyau. Barre: $5 \mu \mathrm{m}$.

$\mathrm{m} / \mathrm{s} n^{\circ} 4$ vol. 10, avmil 94 humains [25]. Une protéine de masse moléculaire élevée $(\geq 350 \mathrm{kDa})$, appartenant au matériel péricentriolaire, interagit fortement avec RlI et est substrat de la PKA. Il est donc vraisemblable que cette protéine centrosomique assure l' "ancrage " de la PKA au niveau du centrosome via sa propriété d'interagir avec la sous-unité régulatrice de cette kinase. Des études de phosphorylation des protéines centrosomiques suggèrent que la PKA pourrait phosphoryler des protéines ayant un rôle de moteur moléculaire.

La cycline Bl est également localisée en partie au centrosome des cellules humaines (figure $4 C$ et $D$ ). Le double marquage avec des anticorps dirigés contre la cycline $\mathrm{Bl}$ et contre un antigène du centrosome (CTR 453) indique une colocalisation des deux antigènes. Cette association n'est pas permanente dans la mesure où la cycline $\mathrm{B} 1$ n'est présente que de la phase $S$ jusqu'à la transition métaphase-anaphase [14], moment où elle est dégradée. Le fait que cette accumulation périodique de cycline $\mathrm{Bl}$ au centrosome coincide parfaitement avec celle de la p34 dtc2 elle-même [26] suggère que la cycline Bl est bien la sousunité régulatrice responsable du ciblage de la p34 $4^{d d 2}$ au centrosome dans les cellules en G2/mitose. Il est à noter que l'autre sous-unité régulatrice de la p34 rds2, la cycline A, interagit aussi avec le centrosome, mais de façon encore plus transitoire: de la prophase à la métaphase [14]. Cette dernière observation soulève l'intéressante possibilité qu'une même sous-unité catalytique puisse être ciblée à différents moments du cycle cellulaire, via différentes sous-unités régulatrices vers différents composants d'un même organite.

Récemment Brewis et al. [27] ont décrit une phosphatase de la famille PP4 (PPX) qui, bien que présente dans le cytoplasme et le noyau, s'accumule au centrosome en interphase et en mitose (figure 4B). La ou les sous-unités régulatrices de la PPX ne sont pas connues actuellement, de sorte qu'il est encore trop tôt pour savoir si la localisation centrosomique de cette phosphatase impli- 
que un mécanisme de ciblage similaire à celui décrit précédemment pour la PPl.

Les conséquences fonctionnelles de toutes ces interactions sont loin d'être élucidées. Cependant, l'utilisation combinée de systèmes acellulaires tels que les extraits d'ovocytes de xénope et de centrosomes isolés de tissus variés ouvre des perspectives très prometteuses quant à l'identification des mécanismes moléculaires mis en jeu pour la localisation subcellulaire de ces différentes kinases ou phosphatases. Ainsi, les résultats récents du groupe de Karsenti [16] indiquent clairement que le ciblage, via les cyclines A et B1, de la même sous-unité catalytique p34 ${ }^{\text {rdc } 2}$ vers différents substrats centrosomiques joue un rôle essentiel dans le contrôle de l'activité de cet organite.

\section{Conclusions et perspectives}

Au sein de la cellule, la spécificité d'action d'un grand nombre de protéine sérine/thréonine kinases et phosphatases semble être assurée par le ciblage de la sous-unité catalytique vers les substrats. La protéine qui dirige la sous-unité catalytique joue également le rôle de régulateur de son activité. Elle est elle-même la cible de multiples autres kinases et phosphatases. Elle permet donc à plusieurs voies de régulation de moduler indirectement l'activité enzymatique du complexe auquel elle est associée. De nombreuses études montrent que l'expression de ces sous-unités de ciblage et leurs interactions privilégiées avec les cibles de ces enzymes semblent jouer un rôle fondamental, non seulement dans la réponse hormonale, mais aussi dans le contrôle de la prolifération cellulaire. Récemment, il a été montré que la modulation de l'expression des différentes unités de ciblage des PKA (en utilisant les stratégies de transfection cellulaire et d'ARN messagers antisens) ouvrirait une perspective thérapeutique de l'hyperprolifération maligne. Il pourrait en être de même dans le cas des unités de ciblage des kinases et des phosphatases impliquées dans le cycle cellulaire

\section{Summary}

Targeting kinases and phosphatases towards specific substrates inside the cell

The broad substrate specificity of most of the kinases and phosphatases, the enzymes involved in the reversible phosphorylation of proteins, must be restricted in vivo to further ensure an efficient and precise signaling. Here we review recent evidence indicating that at least two families of kinases and one family of phosphatases use a similar strategy to target their catalytic subunit towards their specific substrates. The targeting subunit of these modular enzymes could direct the catalytic subunit to their appropriate target locus. The centrosome, the organiser of cell microtubules, is chosen to illustrate a privileged target locus for several kinases and phosphatases, using such a targeting subunit strategy.

\section{Remerciements}

Nous remercions tout particulièrement Michel Bornens dans le laboratoire duquel la majorité du travail rapporté dans cet article a été effectuée. We also acknowledge DrNeil Brewis, Dr Alan Prescott and DrP.W. Cohen for the micrograph, figure $4 B$. Nous tenons également à remercier les Dr Danièle Evain-Brion et Jean-Paul Thiéry pour leurs commentaires sur cette revue.

\section{TIRES A PART}

(x. Keryer. 